3. G. H. Hardy, Notes on some points in the integral calculus, Messenger of Mathematics vol. 58 (1929) pp. 50-52.

4. Einar Hille and J. D. Tamarkin, On summability of Fourier series. II, Ann. of Math. (2) vol. 34 (1933) pp. 329-348.

5. S. Verblunsky, On some classes of Fourier series, Proc. London Math. Soc. vol. 33 (1932) pp. 287-327.

6. F. H. Young, A matrix transformation of Fourier coefficients, Thesis, University of Oregon, 1950.

7. Antoni Zygmund, Trigonometrical series, Warsaw-Lwow, 1935, 331 pp.

UNIVERSITY OF OREGON

\title{
A REMARK ON LINEAR ELLIPTIC DIFFERENTIAL EQUATIONS OF SECOND ORDER
}

\section{EBERHARD HOPF}

Consider a linear partial differential expression

$$
L(u)=\sum_{i, k} a_{i k}(x) \frac{\partial u}{\partial x_{i} \partial x_{k}}+\sum_{i} b_{i}(x) \frac{\partial u}{\partial x_{i}}
$$

with no term $c(x) u$. The coefficients $a_{i k}$ and $b_{i}$ are suppose to be continuous in an open connected set $R$ of $x$-space, $x=\left(x_{1}, \cdots, x_{n}\right)$. Let $x^{0}$ denote a point on the boundary of $R$ which has the property that $R$ contains the interior of a hypersphere $\left|x-x^{*}\right|<r_{0}$ with $x^{0}$ on its boundary. Suppose that the coefficients are continuous at $x=x^{0}$ also. Let, finally, $L$ be elliptic in $R+x^{0}$ such that the quadratic form

$$
\sum a_{i k}(x) \lambda_{i} \lambda_{k}
$$

is positive definite in each point of $R+x^{0}$.

This note contains a simple proof of the following:

Theorem. Suppose that $u=u(x)$ is of class $C^{\prime \prime}$ in $R$ and that $u \geqq 0$, $L(u) \leqq 0$ in $R$. If the limit value of $u$ at $x=x^{0}$ is zero, then either the normal derivative $d u / d n$ at $x=x^{0}$, understood as the limit inferior of $\Delta u / \Delta n$, is $>0$ or $u \equiv 0$ in $R$.

Special cases of the theorem have been known for a long time. It contains, in particular, the fact that Green's function of $L$ has a positive normal derivative along the boundary if the boundary is sufficiently smooth.

To prove the theorem we note first that $u \geqq 0$ in $R$ and $u\left(x^{0}\right)=0$ trivially implies $d u / d n \geqq 0$. The hypotheses that $u \geqq 0$ in $R$ and $L(u)$ $\leqq 0$ in $R$ imply that either $u>0$ in $R$ or $u \equiv 0$ in $R$. This follows from

Received by the editors February 18, 1952. 
the sharp maximum-minimum-theorem. ${ }^{1}$ It suffices to prove that $d u / d n>0$ at $x^{0}$ if $u>0$ in $R$. Consider the sphere mentioned in the hypothesis. It may be assumed that its boundary has no other point in common with the boundary of $R$ than $x^{0}$. Otherwise a second sphere which is internally tangent to the first one at $x=x^{0}$ would satisfy this condition. We choose its center as origin of the coordinatesystem and we set $r=|x|$. Consider the closed spherical shell $S$ : $r_{0} / 2 \leqq r \leqq r_{0}$ where $r_{0}$ denotes the radius of the sphere. ${ }^{2}$ Obviously $u$ is continuous in $S$, and

$$
\begin{aligned}
& u \geqq 0 \quad \text { on } \quad r=r_{0}, \\
& u=0 \quad \text { at the point } x^{0} \text { of } r=r_{0}, \\
& u>0 \quad \text { on } \quad r=r_{0} / 2 .
\end{aligned}
$$

In my proof of the extremum-theorem I considered the auxiliary function

$$
h(x)=e^{-a r^{2}}-e^{-a r_{0}^{2}} .
$$

It has the property that $h>0, r<r_{0}$, and that

$$
L(h)>0, \quad r_{0} / 2<r<r_{0},
$$

if the constant $a$ is chosen sufficiently large. The reader can easily verify this fact himself if he uses the ellipticity of $L$ and the continuity of the coefficients in the closed region $S . h$ is of class $C^{\prime \prime}$ in $S$, and

$$
h=0 \quad \text { on } \quad r=r_{0} .
$$

The function

$$
v=u-\epsilon h,
$$$$
\epsilon>0,
$$

is of class $C^{\prime \prime}$ in the interior of $S$ and continuous in $S$. Moreover, by (1) and (3),

$$
v \geqq 0 \text { on } \quad r=r_{0} \text {. }
$$

1 E. Hopf, Elementare Bemerkungen über die Lösungen partieller Differentialgleichungen zweiter Ordnung vom elliptischen Typus, Sitzungsberichte der Berliner Akademie der Wissenschaften vol. 19 (1927) pp. 147-152.

2 I owe the idea of using this type of region to my colleague D. Gilbarg who used it in a special case in order to prove the uniqueness of free boundary flow under more general conditions than considered hitherto. He considers a special differential equation $L(u)=0$ and uses a special solution $h, L(h)=0$, as an auxiliary function. See his paper Uniqueness of axially symmetric flows with free boundaries, Journal of Rational Mechanics and Analysis vol. 1 (1952) pp. 309-320, in particular pp. 314-315. 
If the constant $\epsilon$ is chosen sufficiently small, then, by the third property (1),

$$
v \geqq 0 \text { also on } r=r_{0} / 2 \text {. }
$$

By hypothesis, $L(u) \leqq 0$ in $S$, and by (2),

$$
L(v)<0, \quad r_{0} / 2<r<r_{0} .
$$

(4), (5), and (6) imply that $v \geqq 0$ holds in the whole of $S$. This follows again from the sharp extremum theorem or, this time more simply, from the more elementary fact that $v$ cannot have a negative minimum in the interior of $S$. But $v \geqq 0$ in $S$ and $v=0$ at $x=x^{0}$ (see (3) and the second property (1)) imply that

$$
\frac{d v}{d n}=\frac{d u}{d n}-\epsilon \frac{d h}{d n} \geqq 0 .
$$

$d h / d n$ is evidently $>0$. Hence $d u / d n>0$, q.e.d.

INDIANA UNIVERSITY 